\title{
1 Proteolytic targeting chimeras with specificity for plasma membrane 2 and intracellular estrogen receptors
}

3

$4 \quad$ Anh S. Lu ${ }^{1 \ddagger}$, Milad Rouhimoghadam ${ }^{2,3}$, Christopher Arnatt ${ }^{4}$, Edward J. Filardo ${ }^{2,3 ¥}$, Aliasger 5 K. Salem ${ }^{1,2 \Psi^{*}}$

6

$7 \quad{ }^{1}$ College of Pharmacy, The University of Iowa, United States, ${ }^{2}$ Holden Comprehensive Cancer

8 Center, University of Iowa Hospitals and Clinics, United States, ${ }^{3}$ Carver College of Medicine, The

9 University of Iowa, United States, ${ }^{4}$ Department of Chemistry, Saint Louis University, St. Louis, 10 MO 63104, USA.

11

12 \$ These authors contributed equally

$13 ¥$ These authors share senior authorship

$14 *$ Corresponding author

15

16

17

18

19

20

21

22

23

24

25

26

27

28

29

30 
31 Supporting Information

32 Table of Contents

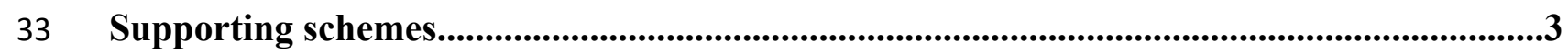

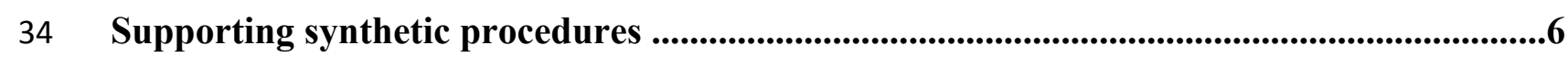

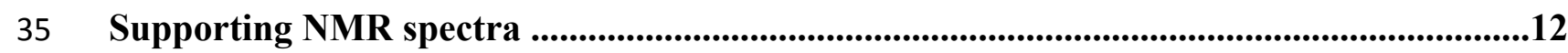

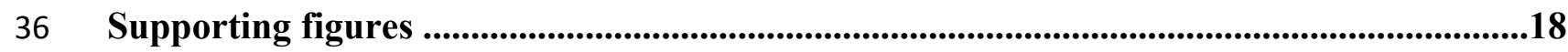

37

38

39

40

41

42

43

44

45

46

47

48

49

50

51

52

53

54

55

56

57

58

59

60

61 
<smiles>CCCCOCCOCCO</smiles><smiles>CCC(=O)OC(C)(C)C</smiles>

67

68

69

70

71

72

73

74

75

76

77

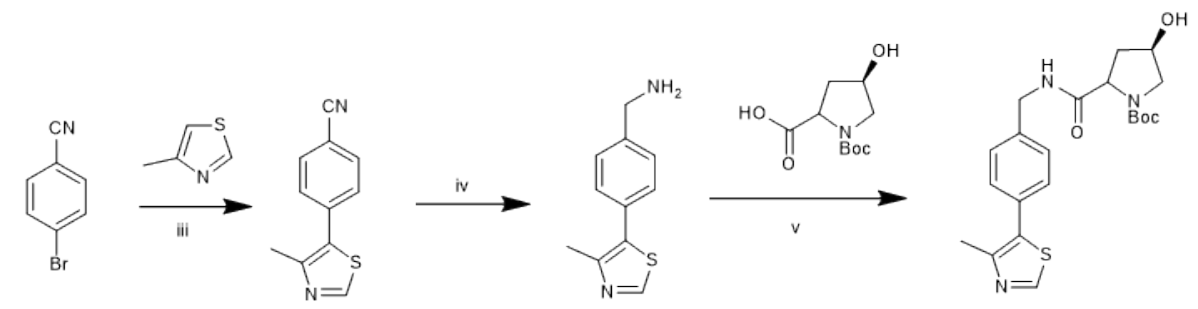

79

80

81

82

3

4<smiles>O=C(O)COCCOCCOCCOCC(=O)O</smiles>

1

3

7

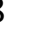

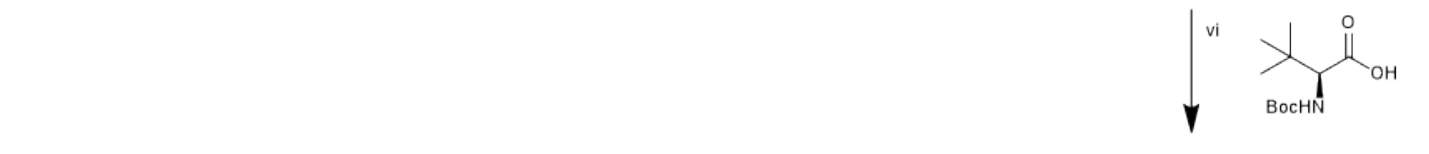

\section{3}
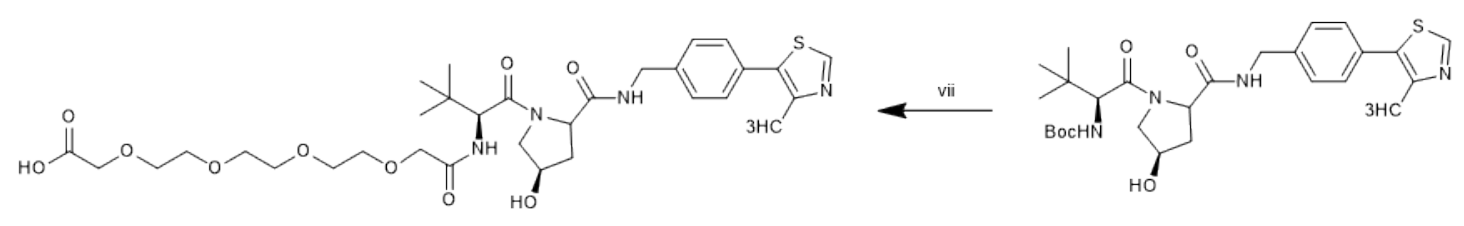

88

89

90

91

92 
94

95

96

97

98

99

100

101

102

103

104

105

106

107

108

109

110

111

112

113

114

115

116

117

118

119

120

121

122

123

124

125

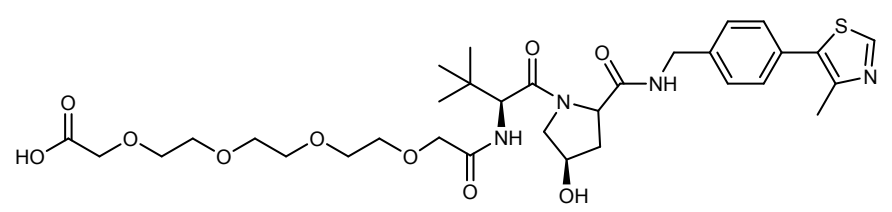

7
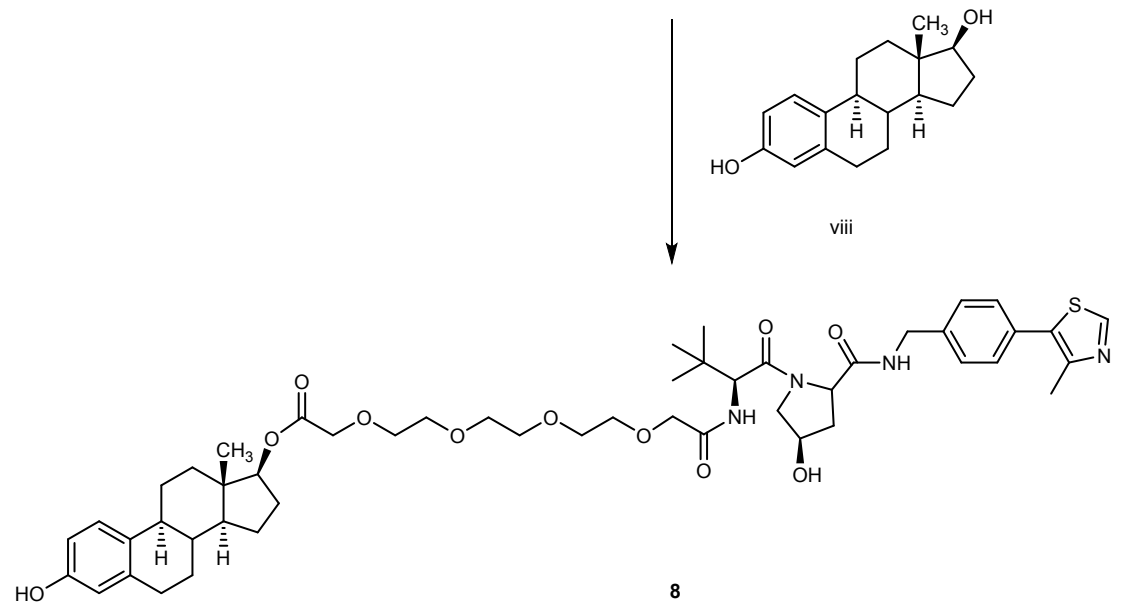

Scheme 2. Synthesis of UI-EP001 (compound 8). Reagents and conditions: (viii) DMAP, EDC, anhydrous DMF, rt, $48 \mathrm{~h}$.

III
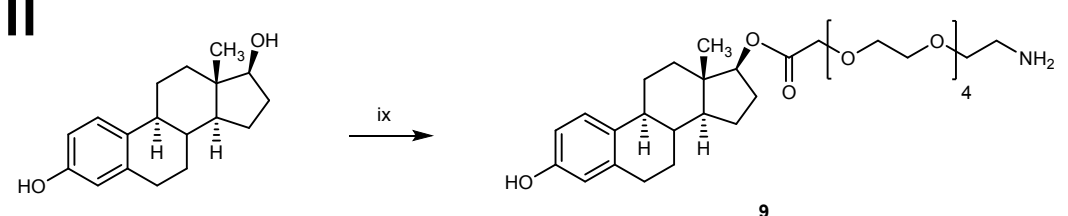

$\downarrow$

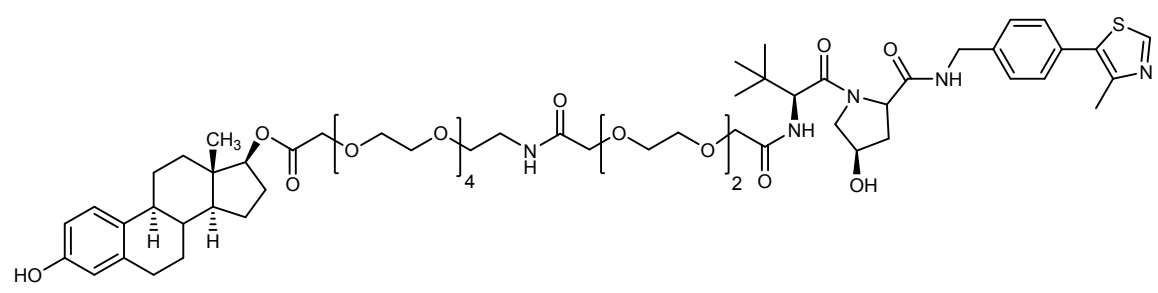

10

Scheme 3. Synthesis of UI-EP002 (compound 10). Reagents and conditions: (ix) FmocNH-PEG ${ }_{8}-\mathrm{CH}_{2} \mathrm{CH}_{2} \mathrm{COOH}$, DMAP, EDC, anhydrous DMF, rt, 72h; (x) 1. Et 3 N, anhydrous DMF; 2. Compound 7, HATU, DIPEA, anhydrous DMF, rt, $48 \mathrm{~h}$. 

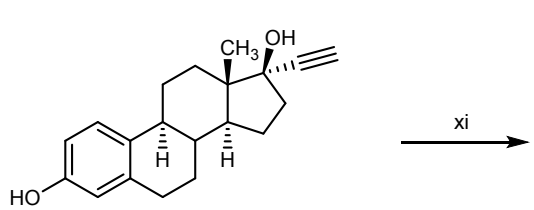

130

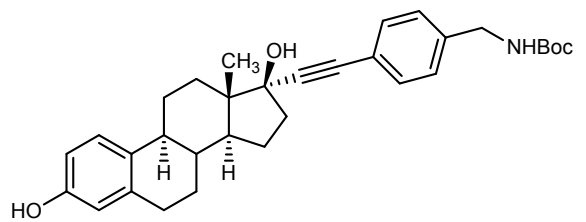

11

131

132

133

134

135

136

137

138

139

140

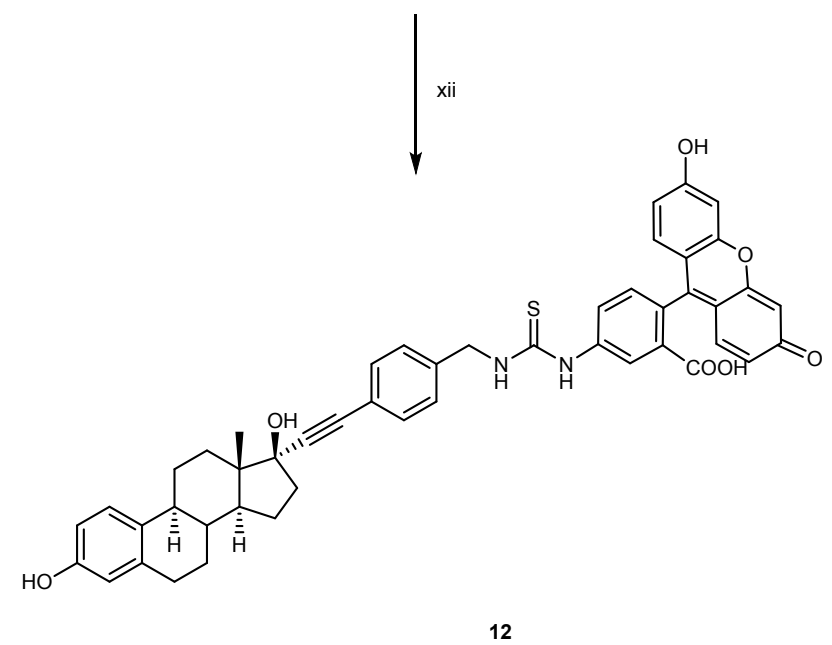

141

142 SI Scheme 4. Synthesis of $E_{2}$-FITC 12. Reagents and conditions: (xi) tert-butyl-4-iodobenzylcarbamate,

$143 \mathrm{Pd}\left(\mathrm{Ph}_{3}\right)_{4}, \mathrm{Cul}, \mathrm{Et}_{3} \mathrm{~N}$, r.t, overnight; (xii) 1. TFA/DCM, rt, 2h; 2. FITC, pyridine, DMF, rt, overnight.

144

145

146

147

148

149

150

151

152

153

154

155

156

157 
161

162

163

164

165

166

167

168

169

170

171

172

173

174

175 176

177

178

179

180

181

182

183

184 185

\section{Supporting synthetic procedures}

\section{Synthesis of UI-EP001 (8).}

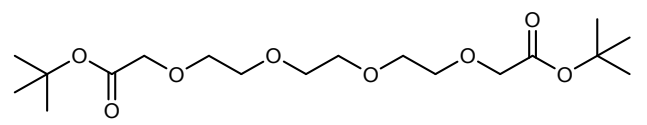

di-tert-butyl 3, 6, 9, 12 - tetraoxatetradecanedioate (1). A mixture of triethylene glycol (1.50 g, $1.36 \mathrm{ml}, 10 \mathrm{mmol}, 1$ eq.), $\mathrm{NaH} 60 \%$ in mineral oil (800 mg, $20 \mathrm{mmol}, 2$ eq.) and tert-Butyl bromoacetate $(4.50 \mathrm{~g}, 3.4 \mathrm{ml}, 20 \mathrm{mmol}, 2$ eq.) were dissolved in $10 \mathrm{~mL}$ of dioxane. Reaction mixture was stirred overnight, followed by quenching with saturated $\mathrm{NH}_{4} \mathrm{Cl}$. The mixture was extracted with EtOAc and dried by $\mathrm{Na}_{2} \mathrm{SO}_{4}$, then concentrated in vacuum. The product was applied to flash chromatography (Hexane:EtOAc $=8: 2$ ) to give compound $\mathbf{1}$ an colorless oil. Yield: 3.25 g, $8.60 \mathrm{mmol}(92 \%) .{ }^{1} \mathrm{H}$ NMR $\left(300 \mathrm{MHz}, \mathrm{CDCl}_{3}\right): \delta 3.99(\mathrm{~s}, 4 \mathrm{H}), 3.72-3.66(\mathrm{~m}, 16 \mathrm{H}), 1.46(\mathrm{~s}$, 9H). HRMS (ESI+) cald for $\mathrm{C}_{18} \mathrm{H}_{34} \mathrm{O}_{8}[\mathrm{M}+1]^{+}: 379.23$, found 379.46.

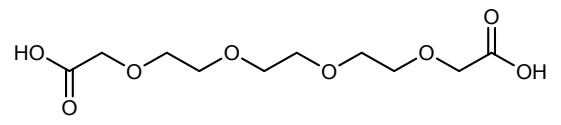

di-tert-butyl 3, 6, 9, 12 - tetraoxatetradecanedioic (2). A solution of compound 1 (1.74 g, 4.62 $\mathrm{mmol})$ in a 50\% v/v trifluoroacetic acid (TFA) in DCM (6 mL per mmol) was stirred at r.t. for 2 h. TLC analysis (10\% methanol in DCM) showed complete conversion of the starting material. Then, the reaction mixture was concentrated under vacuum and the crude product was freeze-dried to obtain the desired product 2 (quantitative yield). ${ }^{1} \mathrm{H}$ NMR $\left(300 \mathrm{MHz}, \mathrm{CDCl}_{3}\right): \delta 4.03(\mathrm{~s}, 4 \mathrm{H})$, 3.85-3.42 (m, 16H). HRMS (ESI+) cald for $\mathrm{C}_{10} \mathrm{H}_{18} \mathrm{O}_{8}[\mathrm{M}+1]^{+}: 267.10$, found 267.56.

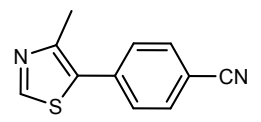

4-(4-Methylthiazol-5-yl) benzonitrile (3). A mixture of 4-bromobenzonitrile (5.00 g, $27.5 \mathrm{mmol})$, 4-methylthiazole (4.98 mL, $54.7 \mathrm{mmol}), \mathrm{KOAc}(5.40 \mathrm{~g}, 55.0 \mathrm{mmol})$, and palladium acetate (62.0 $\mathrm{mg}, 0.27 \mathrm{mmol}$ ) were dissolved in $20 \mathrm{~mL}$ of DMAc. The mixture was heated to $120^{\circ} \mathrm{C}$ overnight, cooled, and diluted with EtOAc. The solution was washed with brine, dried with $\mathrm{Na}_{2} \mathrm{SO}_{4}$ and concentrated. The resulting oil was applied to flash chromatography (Hexane:EtOAc = 9:1 -> 1:1 -> 1:9) . Compound 3 was obtained as a yellow solid (4.49 g, $22.5 \mathrm{mmol}, 82 \%)$. ${ }^{1} \mathrm{H}$ NMR (300 $\left.\mathrm{MHz}, \mathrm{CDCl}_{3}\right) \delta 8.76(\mathrm{~s}, 1 \mathrm{H}), 7.75-7.71(\mathrm{~m}, 2 \mathrm{H}), 7.60-7.55(\mathrm{~m}, 2 \mathrm{H}), 2.58-2.56(\mathrm{~m}, 3 \mathrm{H})$. HRMS (ESI+) cald for $\mathrm{C}_{11} \mathrm{H}_{8} \mathrm{~N}_{2} \mathrm{~S}[\mathrm{M}+1]^{+}:$201.04, found 201.36. 
(4-(4-Methylthiazol-5-yl) phenyl) methanamine (4). Compound 3 (4.49 g, $22.5 \mathrm{mmol}$ ) was dissolved in $300 \mathrm{~mL}$ anhydrous $\mathrm{MeOH}$. Cobalt chloride $\left(\mathrm{CoCl}_{2}\right)$ (4.39g, $\left.33.75 \mathrm{mmol}\right)$ was added and the solution was cooled an ice bath for $30 \mathrm{~min} . \mathrm{NaBH}_{4}(5.22 \mathrm{~g}, 138 \mathrm{mmol})$ was added in fractions over the course of $20 \mathrm{~min}$. The mixture was stirred for another $90 \mathrm{~min}$ and quenched with cold $\mathrm{H}_{2} \mathrm{O}$. The mixture was filtered, diluted in $\mathrm{H}_{2} \mathrm{O}$ and extracted with EtOAc. The organic layer was dried with $\mathrm{Na}_{2} \mathrm{SO}_{4}$, filtered, concentrated, and purified via flash chromatography (DCM:MeOH = 99:1 -> 90:10, with 0.5 $\mathrm{M} \mathrm{Et}_{3} \mathrm{~N}$ ). Compound 4 was obtained as white powder (3.44 g, $16.88 \mathrm{mmol}, 75 \%) .{ }^{1} \mathrm{H}$ NMR (300 MHz, DMSO-d 6 ) $\delta 8.94(\mathrm{~s}, 1 \mathrm{H}), 7.42(\mathrm{~m}, 4 \mathrm{H}), 3.77(\mathrm{~s}, 2 \mathrm{H})$, 2.45 (s, 3H). ${ }^{13} \mathrm{C}$ NMR (300 MHz, DMSO- $\left.d_{6}\right) \delta 152.23,147.98,145.01,131.89,129.95,129.01$, 127.35, 45.23, 15.98. HRMS (ESI+) cald for $\mathrm{C}_{11} \mathrm{H}_{12} \mathrm{~N}_{2} \mathrm{~S}[\mathrm{M}+1]^{+}:$205.07, found 205.77.

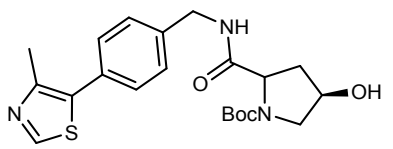

(4R)-4-Hydroxy-N-(4-(4-methylthiazol-5-yl) benzyl)-Boc-pyrrolidine-2-carboxamide (5). A mixture of compound 4 (2.04 g, $10.00 \mathrm{mmol})$, Boc-Hyp-OH (2.31 g, $10.00 \mathrm{mmol})$, DIPEA (6.95 $\mathrm{mL}, 40.00 \mathrm{mmol})$, and HBTU $(4.16 \mathrm{~g}, 11.00 \mathrm{mmol})$ were dissolved in $50 \mathrm{~mL}$ of anhydrous DMF. The mixture was stirred at room temperature overnight, then diluted with $\mathrm{H}_{2} \mathrm{O}$ and extracted with EtOAc. The organic layer was washed with brine, dried with $\mathrm{Na}_{2} \mathrm{SO}_{4}$, and concentrated. The crude product was purified via flash chromatography (DCM:MeOH =99:1 -> 90:10). Compound 5 was obtained as colorless oil (2.79 g, $6.7 \mathrm{mmol}, 67 \%){ }^{1} \mathrm{H}$ NMR $\left(300 \mathrm{MHz}, \mathrm{CDCl}_{3}\right) \delta 8.69(\mathrm{~s}, 1 \mathrm{H}), 8.28$ - 8.04 (m, 1H), 7.45 - 7.32 (m, 4H), 4.52 - 4.44 (m, 3H), 4.10 (t, 1H), $3.10-3.00$ (m, 1H), 2.88 $2.77(\mathrm{~m}, 1 \mathrm{H}), 2.54(\mathrm{~s}, 3 \mathrm{H}), 2.41-2.32(\mathrm{~m}, 1 \mathrm{H}), 2.07-1.93(\mathrm{~m}, 1 \mathrm{H}), 1.43(\mathrm{~s}, 9 \mathrm{H}) .{ }^{13} \mathrm{C}$ NMR $(300$ $\left.\mathrm{MHz}, \mathrm{CDCl}_{3}\right) \delta 172.15,153.88,152.12,148.02,139.75,131.52,130.66,129.05,128.05,77.25$, 65.48, 55.98, 53.95, 42.16, 28.96, 14.86. HRMS (ESI+) cald for $\mathrm{C}_{21} \mathrm{H}_{27} \mathrm{~N}_{3} \mathrm{O}_{4} \mathrm{~S}[\mathrm{M}+1]^{+}: 418.17$, found 418.03 .

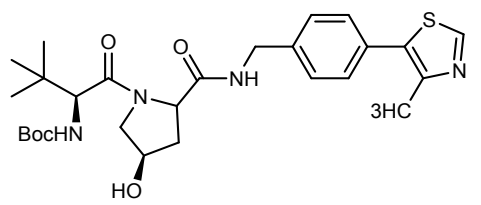

(4R)-1-((S)-2-Boc-Amino-3,3-dimethylbutanoyl)-4-hydroxy-N-(4-(4-methylthiazol-5-yl) benzyl) pyrrolidine-2-carboxamide (6). Compound 5 (2.79, $6.7 \mathrm{mmol})$ was dissolved in 50\% v/v 
TFA/DCM $(6 \mathrm{~mL}$ per mmol $)$ and stirred at room temperature for $2 \mathrm{~h}$. The mixture was concentrated and used for next step without further purification. A mixture of last step product, Boc-L-tertleucine (1.54 g, $6.7 \mathrm{mmol})$, HBTU (2.79 g, $7.37 \mathrm{mmol})$, and DIPEA (4.66 mL, $26.8 \mathrm{mmol})$ was dissolved in $50 \mathrm{~mL}$ of DMF. The mixture was stirred at room temperature overnight, diluted with $\mathrm{H}_{2} \mathrm{O}$, and extracted with EtOAc. The organic layer was washed with a saturated $\mathrm{NaHCO}_{3}$ solution, brine, dried with $\mathrm{Na}_{2} \mathrm{SO}_{4}$, filtered, and concentrated. Crude product was purified with flash chromatography (DCM:MeOH = 99:1 -> 90:10), giving compound 6 as white powder (2.09 g, $3.95 \mathrm{mmol}, 59 \%$ yield after two steps). ${ }^{1} \mathrm{H}$ NMR (300 MHz, DMSO- $\left.d_{6}\right) \delta 8.98(\mathrm{~s}, 1 \mathrm{H}), 7.56-7.50$ (m, 4H), $4.91-4.86(\mathrm{~m}, 4 \mathrm{H}), 4.08(\mathrm{~s}, 1 \mathrm{H}), 3.36-3.31(\mathrm{~m}, 1 \mathrm{H}), 2.58(\mathrm{~s}, 3 \mathrm{H}), 2.34-2.30(\mathrm{~m}, 1 \mathrm{H})$, $2.13-2.07(\mathrm{~m}, 1 \mathrm{H}), 1.20-1.02(\mathrm{~m}, 18 \mathrm{H}) .{ }^{13} \mathrm{C}$ NMR $\left(300 \mathrm{MHz}, \mathrm{DMSO}-d_{6}\right) \delta$ 173.12, 170.15, 157.23, 153.48, 148.16, 139.68, 132.36, 130.12, 128.79, 125.56, 75.69, 65.26, 58.56, 55.23, 53.29, 42.56, 38.26, 36.18, 27.48, 25.42, 15.99. HRMS (ESI+) cald for $\mathrm{C}_{27} \mathrm{H}_{38} \mathrm{~N}_{4} \mathrm{O}_{5} \mathrm{~S}[\mathrm{M}+1]^{+}:$: 531.26, found 531.49 .

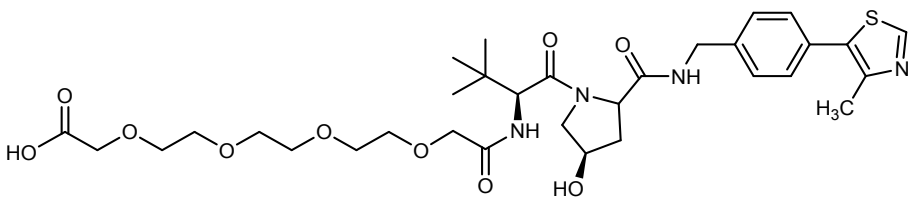

Partial PROTAC (7). Compound $5(2.09,3.95 \mathrm{mmol})$ was dissolved in $50 \% \mathrm{v} / \mathrm{v}$ TFA/DCM (6 mL per mmol) and stirred at room temperature for $2 \mathrm{~h}$. The mixture was concentrated and used for next step without further purification. A mixture of last step product (1 eq), compound 2 ( $2.1 \mathrm{~g}$, $7.9 \mathrm{mmol}, 2 \mathrm{eq})$, HBTU (1.65 g, $4.30 \mathrm{mmol})$, and DIPEA (2.75 mL, $15.8 \mathrm{mmol})$ was dissolved in $50 \mathrm{~mL}$ of DMF. The mixture was stirred at room temperature overnight. As TLC showed complete reaction of starting material, the mixture was diluted in DCM and washed with brine, dried and concentrated. The product was purified by HPLC reverse phase column (Zorbax300SB-C18, 21.2x150mm, 5uCrt) to obtained desire product 7. Yield: (1.21 g, $46 \%$ after 2 steps). ${ }^{1} \mathrm{H}$ NMR $\left(500 \mathrm{MHz}, \mathrm{MeOD}-d_{4}\right) \delta 9.68(\mathrm{~s}, 1 \mathrm{H}), 7.56-7.50(\mathrm{~m}, 4 \mathrm{H}), 4.67$ - $4.69(\mathrm{t}, 1 \mathrm{H}), 4.58$ - $4.55(\mathrm{~m}$, 2H), $4.43-4.40(\mathrm{~d}, 1 \mathrm{H}), 4.15-4.11(\mathrm{~m}, 4 \mathrm{H}), 4.08-4.05(\mathrm{~m}, 17 \mathrm{H}), 3.78-3.80(\mathrm{~m}, 1 \mathrm{H}), 3.85-$ $3.71(\mathrm{~m}, 1 \mathrm{H}), 3.36-3.31(\mathrm{~m}, 1 \mathrm{H}), 2.58(\mathrm{~s}, 3 \mathrm{H}), 2.34-2.30(\mathrm{~m}, 1 \mathrm{H}), 2.13-2.07(\mathrm{~m}, 1 \mathrm{H}), 1.20-$ $1.02(\mathrm{~m}, 9 \mathrm{H}) .{ }^{13} \mathrm{C}$ NMR $\left(500 \mathrm{MHz}, \mathrm{MeOD}-d_{4}\right) \delta 172.97,170.73,170.31,151.43,147.69,138.87$, $132.01,130.15,129.00,127.58,70.93,70.37,70.24,70.20,69.77,69.73,69.66,59.41,56.75$, $56.69,50.39,42.33,37.53,37.70,29.26,25.56,14.40$. HRMS (ESI+) cald for $\mathrm{C}_{31} \mathrm{H}_{44} \mathrm{~N}_{4} \mathrm{O}_{10} \mathrm{~S}[\mathrm{M}$ $+1]^{+}: 679.29$, found 679.89 . 
243

244

245

246

247

248

249

250

251

252

253

254

255

256

257

258

259

260

261

262

263

264

265

266

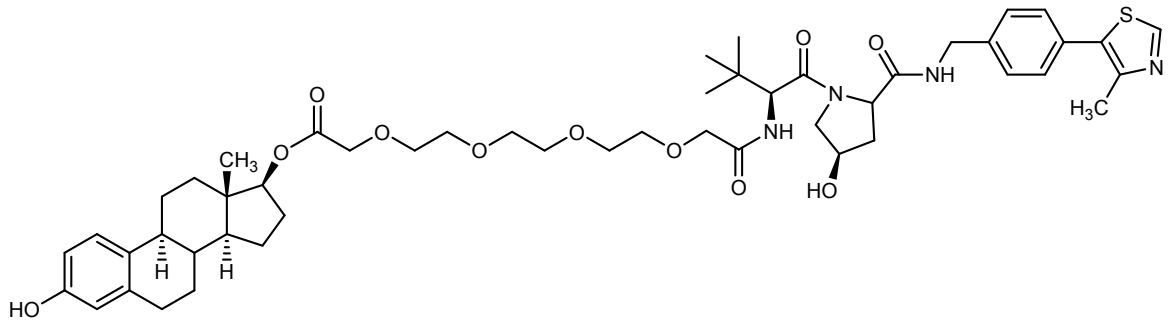

UI-EP001 (8). Compound 7 (300 mg, $0.46 \mathrm{mmol})$, was activated by $N$-Hydroxysuccinimide (NHS) $(53 \mathrm{mg}, 0.46 \mathrm{mmol})$ in $5 \mathrm{~mL}$ of DMF for $3 \mathrm{~h}$, followed by the addition of $17 \beta$-estradiol (125 mg, $0.46 \mathrm{mmol}$ ) and 1-ethyl-3-(3-dimethylaminopropyl)carbodiimide (EDC) (71.3 $\mathrm{mg}, 0.46 \mathrm{mmol})$. Reaction mixture was stirred at room temperature for $48 \mathrm{~h}$ and monitored by reverse phase HPLC. The crude product was diluted in DCM:MeOH (90:10) and washed with brine, dried and concentrated. The product $\mathbf{8}$ was obtained by HPLC reverse phase column (Zorbax300SB-C18, 21.2x150mm, 5uCrt). Yield (119 mg, 28\%). ${ }^{1} \mathrm{H}$ NMR (500 MHz, MeOD- $\left.d_{4}\right) \delta 9.64(\mathrm{~s}, 1 \mathrm{H}), 7.56$ - $7.49(\mathrm{~m}, 4 \mathrm{H}), 7.09-7.07$ (d, 1H), 6.55 - $6.53(\mathrm{~m}, 1 \mathrm{H}), 6.48-6.49(\mathrm{~d}, 1 \mathrm{H}), 4.71-4.67(\mathrm{t}, 1 \mathrm{H})$, 4.58 - $4.55(\mathrm{~m}, 2 \mathrm{H}), 4.12-3.95(\mathrm{~m}, 17 \mathrm{H}), 3.86-3.84(\mathrm{~d}, 1 \mathrm{H}), 3.74-3.71(\mathrm{~m}, 1 \mathrm{H}), 3.68-3.65(\mathrm{t}$, 1H), $2.78-2.77(\mathrm{~m}, 2 \mathrm{H}), 2.57(\mathrm{~s}, 3 \mathrm{H}), 2.32-2.30(\mathrm{~m}, 2 \mathrm{H}), 2.14-1.95(\mathrm{~m}, 4 \mathrm{H}), 1.74-1.67(\mathrm{~m}$, 1H), $1.54-1.18(\mathrm{~m}, 7 \mathrm{H}), 1.15(\mathrm{~s}, 9 \mathrm{H}), 0.78(\mathrm{~m}, 3 \mathrm{H}) .{ }^{13} \mathrm{C} \mathrm{NMR}\left(500 \mathrm{MHz}, \mathrm{MeOD}-d_{4}\right) \delta 173.12$, $170.15,157.23,153.48,148.16,139.68,132.36,130.12,128.79,125.56,75.69,65.26,58.56$, 55.23, 53.29, 42.56, 38.26, 36.18, 27.48, 25.42, 15.99. HRMS (ESI+) cald for $\mathrm{C}_{50} \mathrm{H}_{68} \mathrm{~N}_{4} \mathrm{O}_{11} \mathrm{~S}[\mathrm{M}$ $+1]^{+}$: 933.46, found 933.23.

\section{Synthesis of UI-EP002 (10).}

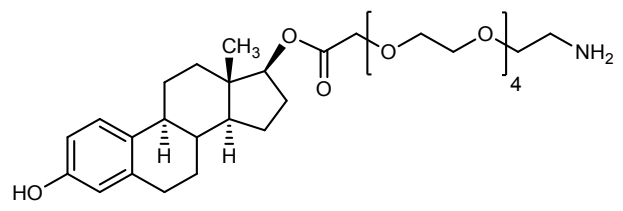

Compound (9). Fmoc-NH-PEG8- $\mathrm{CH}_{2} \mathrm{CH}_{2} \mathrm{COOH}(300 \mathrm{mg}, 0.3 \mathrm{mmol}$ ) was activated by $N$ Hydroxysuccinimide (NHS) (34.5 mg, $0.3 \mathrm{mmol})$ in $1 \mathrm{~mL}$ of DMF for $3 \mathrm{~h}$, followed by the addition of $17 \beta$-estradiol ( $81.6 \mathrm{mg}, 0.3 \mathrm{mmol}$ ) and EDC (46.6 mg, $0.3 \mathrm{mmol}$ ). Reaction mixture was stirred at room temperature for $72 \mathrm{~h}$ and monitored by reverse phase HPLC. Crude product was purified by HPLC reverse phase column (Zorbax300SB-C18, 21.2x150mm, 5uCrt) to give compound 9 Yield (41 mg, 16\%). ${ }^{1} \mathrm{H}$ NMR (500 MHz, MeOD- $\left.d_{4}\right) \delta 7.12-7.11(\mathrm{~d}, 1 \mathrm{H}), 6.59-6.57(\mathrm{~m}, 1 \mathrm{H})$, 
275

6.52-6.51 (d, 1H), $4.26-4.24(\mathrm{~m}, 1 \mathrm{H}), 3.77-3.74(\mathrm{t}, 2 \mathrm{H}), 3.71-3.56(\mathrm{~m}, 31 \mathrm{H}), 3.34-3.32(\mathrm{~m}$, 2H), $2.83-2.80(\mathrm{~m}, 2 \mathrm{H}), 2.59-2.56(\mathrm{t}, 2 \mathrm{H}), 2.36-2.31(\mathrm{~m}, 1 \mathrm{H}), 2.17-2.14(\mathrm{~m}, 1 \mathrm{H}), 2.09-$ $2.05(\mathrm{~m}, 1 \mathrm{H}), 2.01-1.97(\mathrm{~m}, 1 \mathrm{H}), 1.92-1.88(\mathrm{~m}, 1 \mathrm{H}), 1.59-1.19(\mathrm{~m}, 8 \mathrm{H}), 0.81(\mathrm{~s}, 3 \mathrm{H}) .{ }^{13} \mathrm{C}$ NMR (500 MHz, MeOD- $\left.d_{4}\right) \delta 171.62,152.12,141.58,138.85,135.04,128.85,125.00,123.42$, $122.38,117.17,112.27,109.95,78.73,67.76,67.74,67.67,67.59,67.53,67.14,64.07,63.81$, $47.54,40.59,38.02,36.76,34.26,32.13,26.94,26.93,26.88,24.75,23.83,20.25,7.90 \mathrm{HRMS}$ (ESI+) cald for $\mathrm{C}_{51} \mathrm{H}_{69} \mathrm{NO}_{13}[\mathrm{M}+1]^{+}:$905.11, found 905.19.

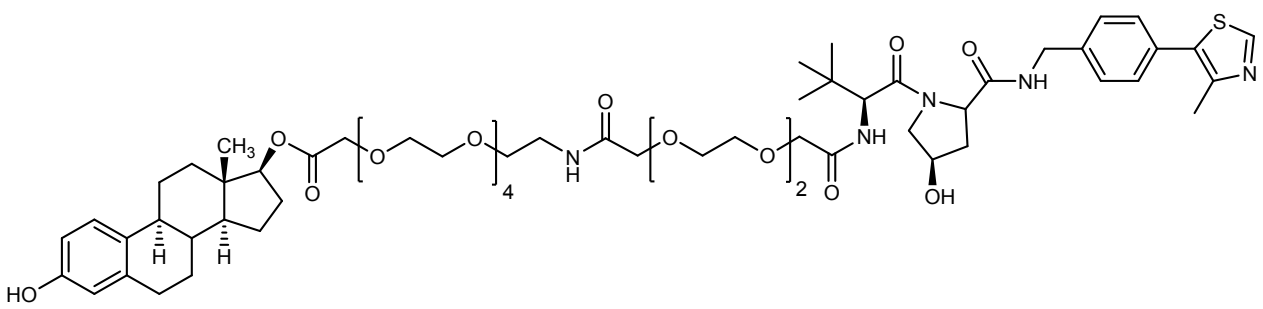

UI-EPOO2 (10). The Fmoc group of Compound $9(35.0 \mathrm{mg}, 0.39 \mathrm{mmol})$ was removed by $\mathrm{Et}_{3} \mathrm{~N}(1$ $\mathrm{mL})$ in DMF $(5 \mathrm{~mL})$. $\mathrm{Et}_{3} \mathrm{~N}$ was removed from the reaction mixture by rotavap, followed by the addition of the partial PROTAC 7 (264.4 mg, $0.39 \mathrm{mmol})$, HATU (178.6 mg, $0.47 \mathrm{mmol}$ ) and DIPEA $(0.271 \mathrm{~mL}, 1.56 \mathrm{mmol})$. Reaction mixture was stirred at room temperature for $48 \mathrm{~h}$ and monitored by reverse phase HPLC. The product 10 was obtained by HPLC reverse phase column (Zorbax300SB-C18, 21.2x150mm, 5uCrt). Yield (26 mg, 52\%). ${ }^{1} \mathrm{H}$ NMR (500 MHz, MeOD- $\left.d_{4}\right)$ $\delta 9.69(\mathrm{~s}, 1 \mathrm{H}), 7.57-7.51(\mathrm{~m}, 4 \mathrm{H}), 7.12-7.11(\mathrm{~d}, 1 \mathrm{H}), 6.59-6.57(\mathrm{~m}, 1 \mathrm{H}), 6.52-6.51(\mathrm{~d}, 1 \mathrm{H})$, $4.71-4.68(\mathrm{~m}, 1 \mathrm{H}), 4.59-4.56(\mathrm{~d}, 1 \mathrm{H}), 4.44-4.40(\mathrm{~d}, 1 \mathrm{H}), 4.26-4.24(\mathrm{~m}, 1 \mathrm{H}), 4.09(\mathrm{~s}, 1 \mathrm{H})$, $3.88-3.86(\mathrm{~m}, 1 \mathrm{H}), 3.77-3.74(\mathrm{t}, 2 \mathrm{H}), 3.71-3.56(\mathrm{~m}, 47 \mathrm{H}), 3.36-3.32(\mathrm{~m}, 6 \mathrm{H}), 2.83-2.23$ (m, 2H), $2.59-2.56(\mathrm{~m}, 5 \mathrm{H}), 2.36-2.31(\mathrm{~m}, 1 \mathrm{H}), 2.21-2.14(\mathrm{~m}, 1 \mathrm{H}), 2.10-2.03(\mathrm{~m}, 1 \mathrm{H}), 2.01$ - $1.97(\mathrm{~m}, 1 \mathrm{H}), 1.92-1.88(\mathrm{~m}, 1 \mathrm{H}), 1.76-1.70(\mathrm{~m}, 1 \mathrm{H}), 1.43-1.30(\mathrm{~m}, 8 \mathrm{H}), 1.15$ (s, 9H), 0.81 (s, 3H). ${ }^{13} \mathrm{C}$ NMR $\left(500 \mathrm{MHz}, \mathrm{MeOD}-d_{4}\right) \delta 174.01,167.15,154.52,143.97,141.25,137.43$, $131.25,127.39,126.77,125.81,124.81,124.78,119.56,114.67,112.34,81.12,70.16,70.13$, 69.98, 69.93, 69.54, 66.47, 66.21, 49.94, 42.98, 40.41, 39.16, 34.52, 29.33, 29.32, 29.17, 27.14, 26.22, 22.64, 10.30. HRMS (ESI+) cald for $\mathrm{C}_{68} \mathrm{H}_{103} \mathrm{~N}_{5} \mathrm{O}_{20} \mathrm{~S}[\mathrm{M}+1]^{+}: 1342.69$, found 1342.15. 


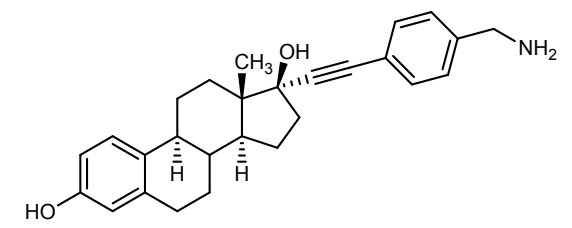

Compound (11). 17a-ethynylestradiol (592 mg, $2 \mathrm{mmol})$ was added to a mixture of 4-(tbutyloxycarbonylaminomethyl)iodobenzene $(778 \mathrm{mg}, 2.33 \mathrm{mmol})$, tetrakis(triphenylphosphine)palladium $\mathrm{Pd}\left(\mathrm{Ph}_{3}\right)_{4}\left(5\right.$ mole \%), and $\mathrm{CuI}\left(5\right.$ mole \%) in $\mathrm{Et}_{3} \mathrm{~N}(20 \mathrm{ml})$ under argon atmosphere. The reaction mixture was stirred at room temperature overnight, then solvent was reduced under vacuum. Crude product was purified by flash chromatography (Hexane:EtOAc = 20:80 -> DCM:MeOH = 90:10) giving yellow powder product. Yield (756 mg, 78\%). ${ }^{1} \mathrm{H}$ NMR (300 MHz,

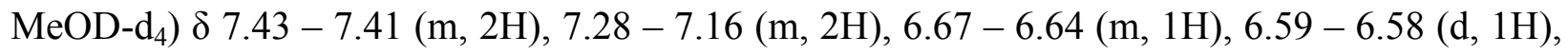
$4.71-4.67(\mathrm{t}, 1 \mathrm{H}), 4.33-4.31(\mathrm{~d}, 1 \mathrm{H}), 2.83(\mathrm{~m}, 2 \mathrm{H}), 2.43-1.72(\mathrm{~m}, 15 \mathrm{H}), 1.57-1.38(\mathrm{~s}, 9 \mathrm{H})$, $0.94(\mathrm{~s}, 3 \mathrm{H}) .{ }^{13} \mathrm{C} \mathrm{NMR}\left(500 \mathrm{MHz}, \mathrm{MeOD}-d_{4}\right) \delta 155.12,143.25,131.59,130.98,125.16,124.33$, 113.25, 113.21, 95.23, 84.03, 77.69, 62.22, 49.96, 45.11, 42.68, 35.66, 33.22, 27.22, 26.45, 25.56, 24.21, 13.56. HRMS (ESI+) cald for $\mathrm{C}_{32} \mathrm{H}_{39} \mathrm{NO}_{3}[\mathrm{M}+1]^{+}:$: 486.29, found 486.85 .

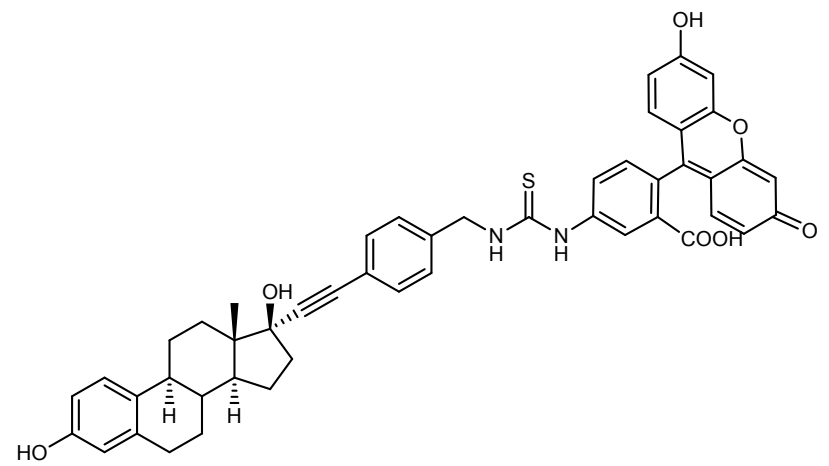

E2-FITC (12). A solution of compound 11 (485 mg, $1 \mathrm{mmol})$ in 50\% v/v TFA/DCM was stirred at r.t. for $2 \mathrm{~h}$. After TLC showed complete conversion, the mixture was co-evaporated with DCM 5-6 times to remove TFA. The residue was redissolved in DMF $(5 \mathrm{~mL})$, followed by the addition of FITC (429 mg, $1.1 \mathrm{mmol})$ and pyridine $(0.25 \mathrm{~mL})$. The reaction mixture was stirred in dark at room temperature overnight. After drying, the crude product was purified by flash chromatography $\left(\mathrm{CHCl}_{3}: \mathrm{MeOH}=95: 5\right.$ - $>$ 90:10) to afford compound 12 as yellow oil (355 mg, 45\%). ${ }^{1} \mathrm{H}$ NMR (300 MHz, MeOD-d4) $\delta 10.23(\mathrm{~s}, 1 \mathrm{H}), 8.05(\mathrm{~m}, 1 \mathrm{H}), 7.83-7.79(\mathrm{~m}, 1 \mathrm{H}), 7.43-7.41(\mathrm{~m}, 2 \mathrm{H})$, $7.27(\mathrm{~m}, 2 \mathrm{H}), 6.68-6.53(\mathrm{~m}, 8 \mathrm{H}), 4.70(\mathrm{t}, 1 \mathrm{H}), 4.32(\mathrm{~m}, 1 \mathrm{H}), 2.85(\mathrm{~m}, 2 \mathrm{H}), 2.40-1.53(\mathrm{~m}, 15 \mathrm{H})$, $0.93(\mathrm{~s}, 3 \mathrm{H})$. HRMS (ESI+) cald for $\mathrm{C}_{48} \mathrm{H}_{42} \mathrm{~N}_{2} \mathrm{O}_{7} \mathrm{~S}[\mathrm{M}+1]^{+}:$791.27, found 791.28. 
319

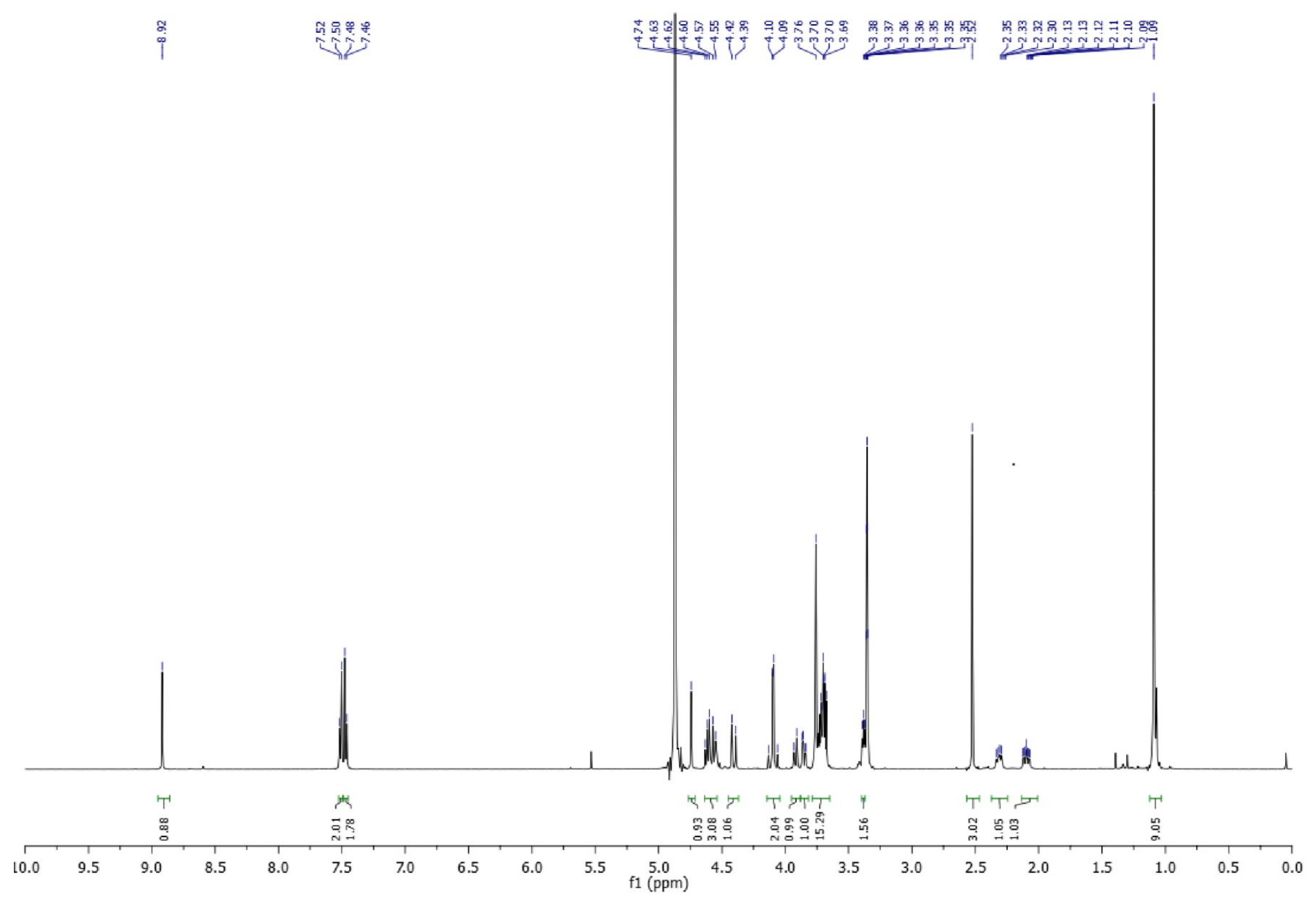




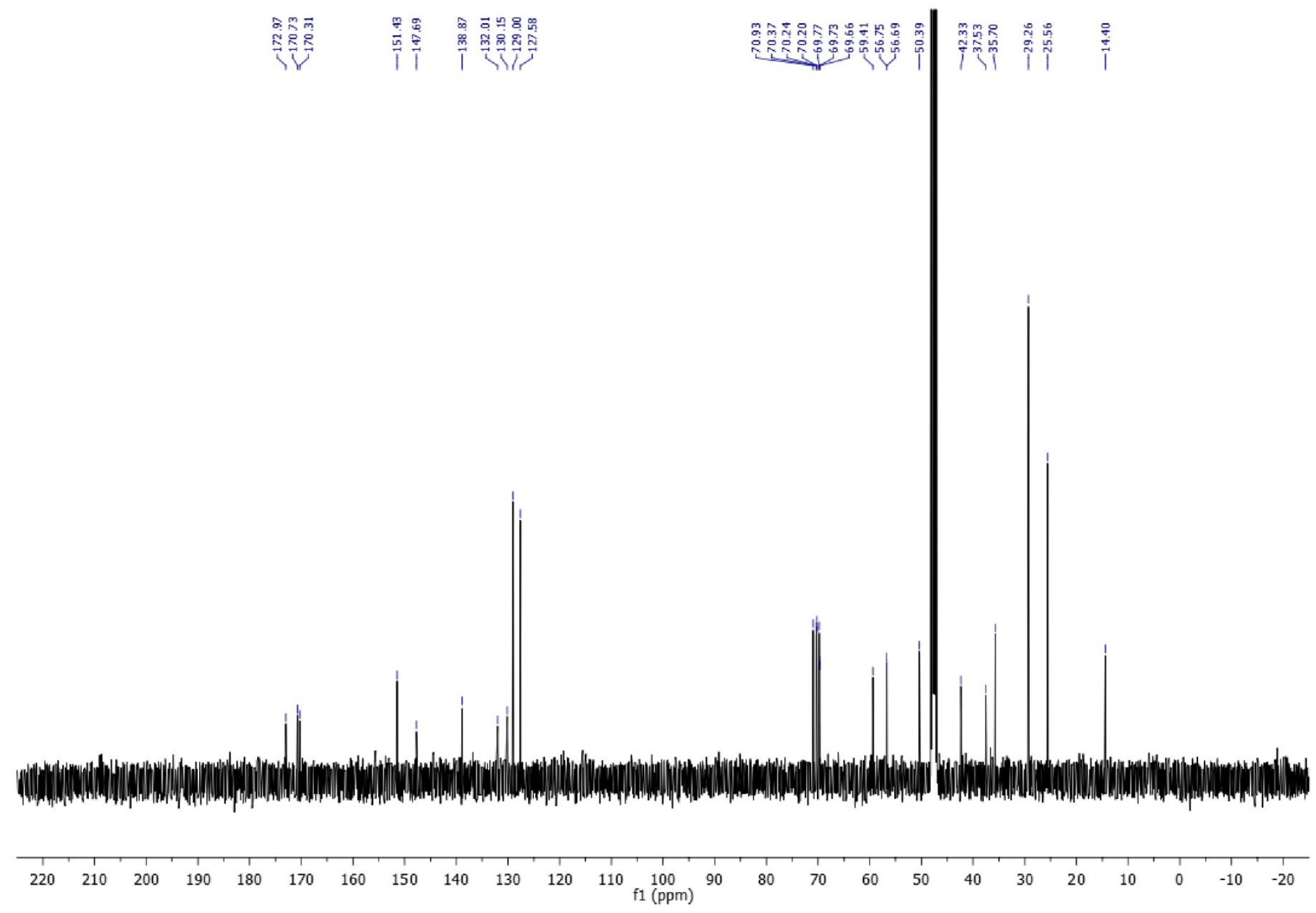


325

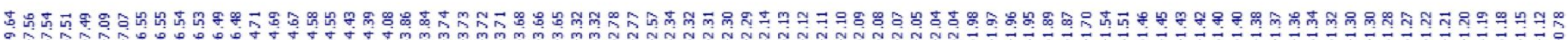

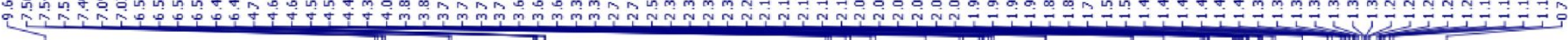

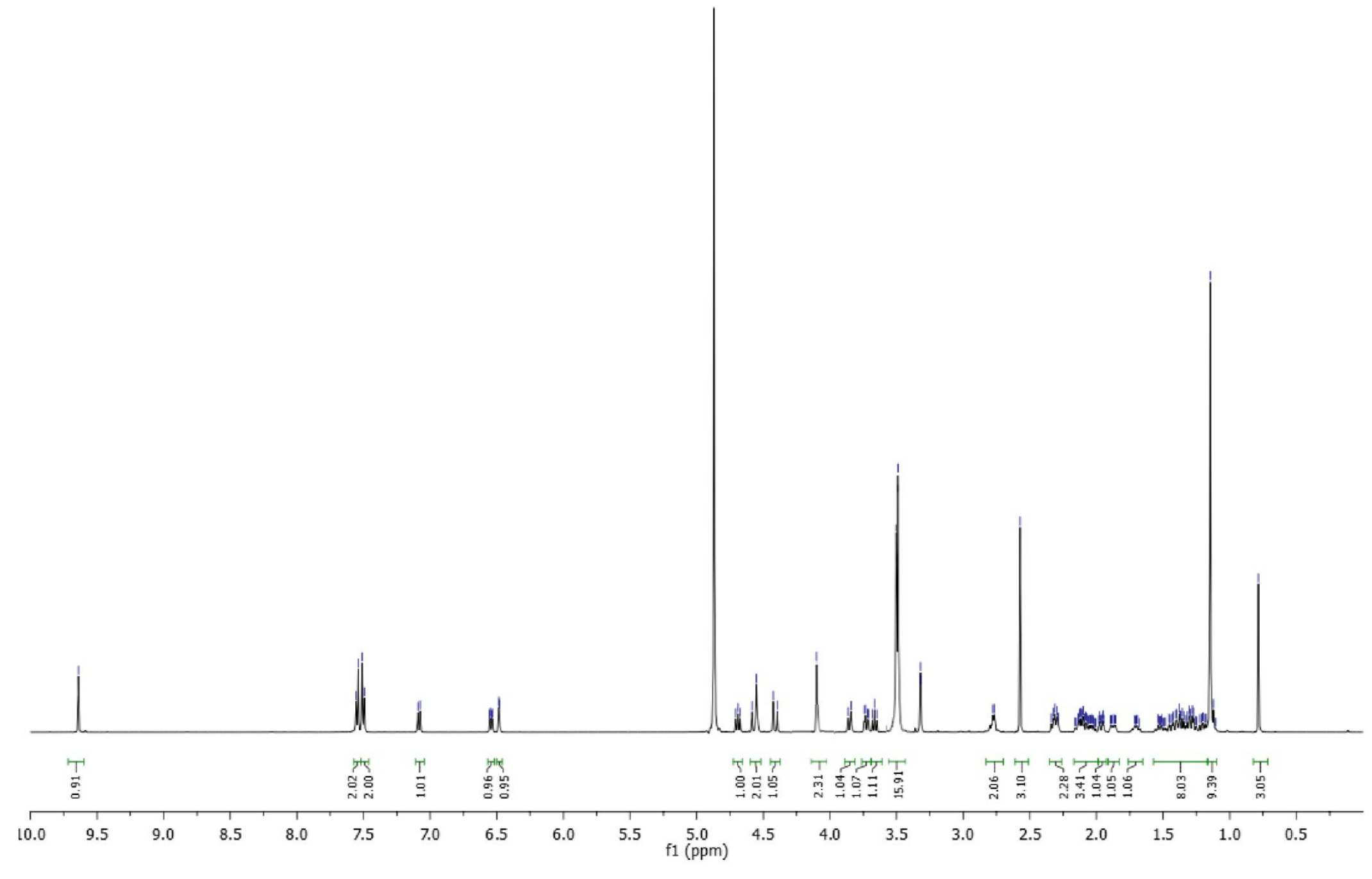


Figure SI 4. UI-EP001 ${ }^{13} \mathrm{C}-\mathrm{NMR}(500 \mathrm{MHz}, \mathrm{MeOD})$

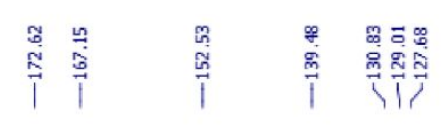

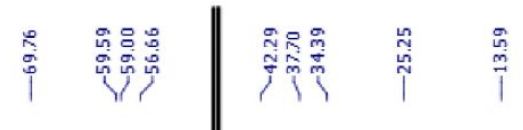

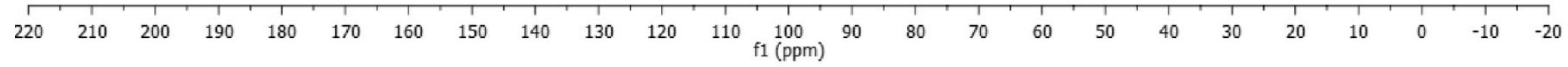




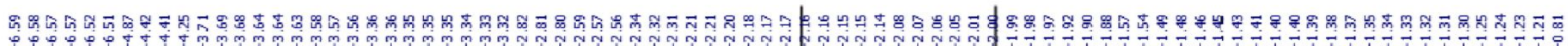

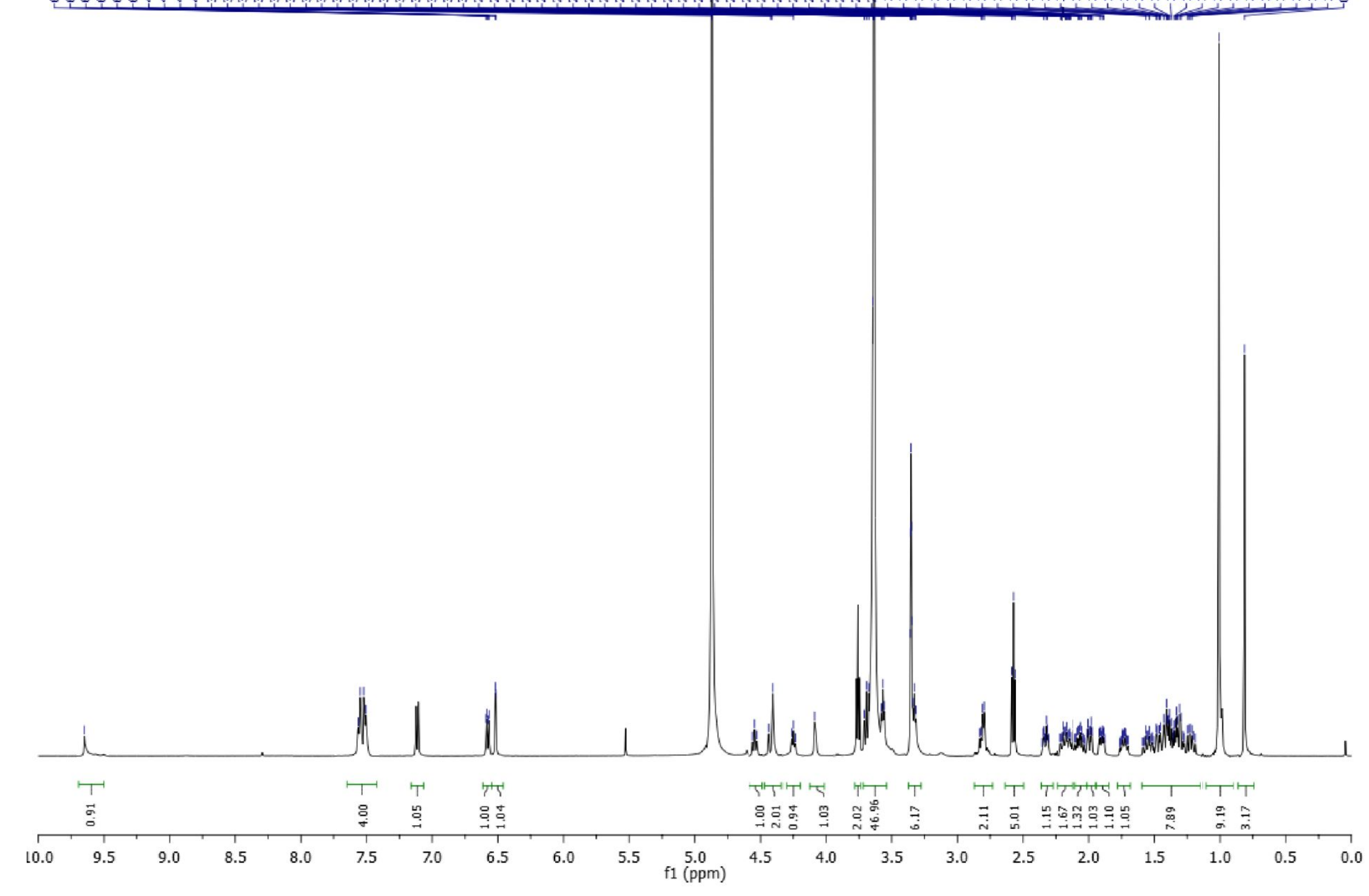


Figure SI 6. UI-EP002 ${ }^{13} \mathrm{C}-\mathrm{NMR}(500 \mathrm{MHz}, \mathrm{MeOD})$

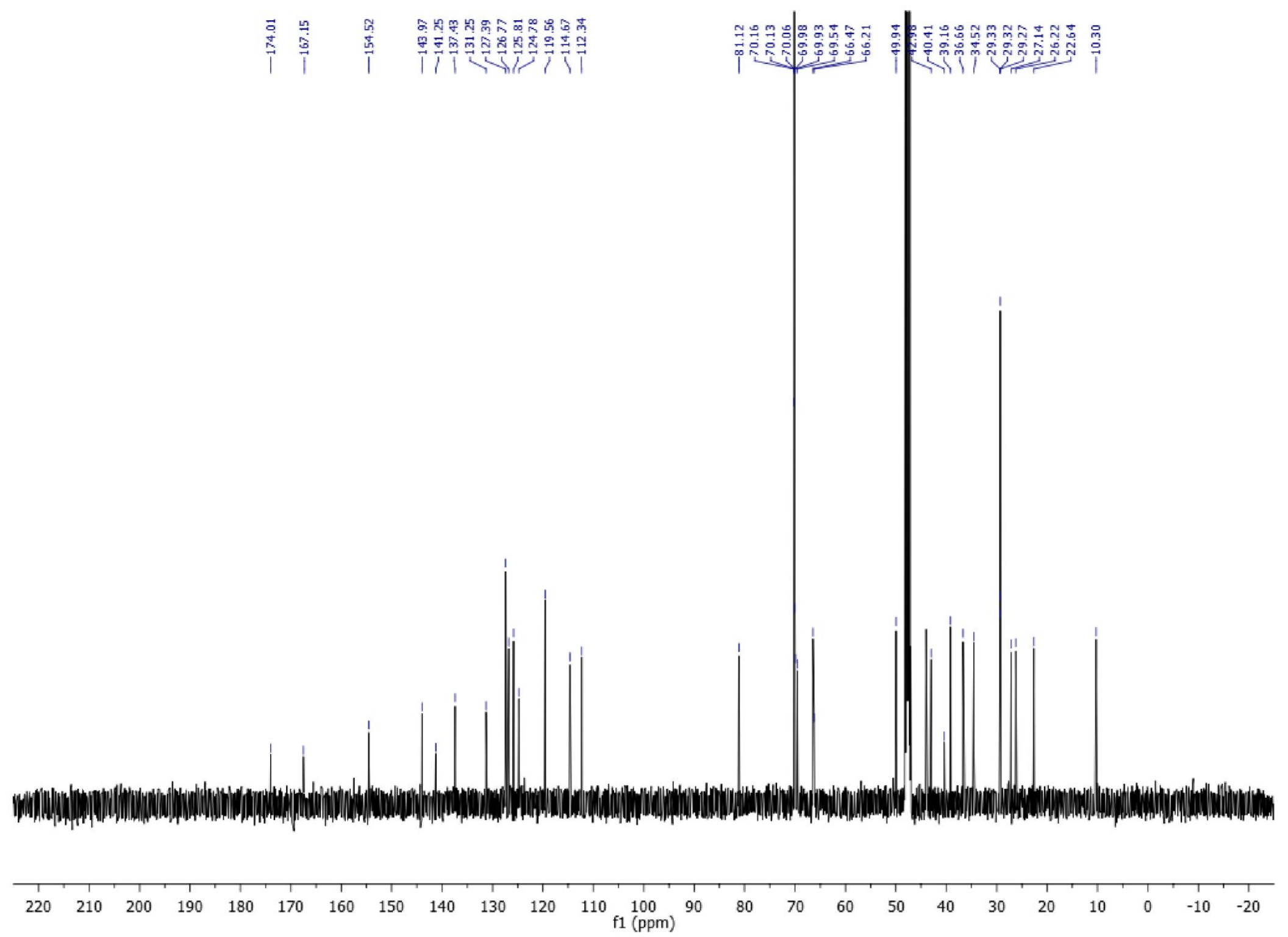


336

337

338

339

340

341

342

343

344

345

346

347

348

349

350

351

352

353

354

355

356

357

358

359

360

361

362

363

\section{Supporting figure}
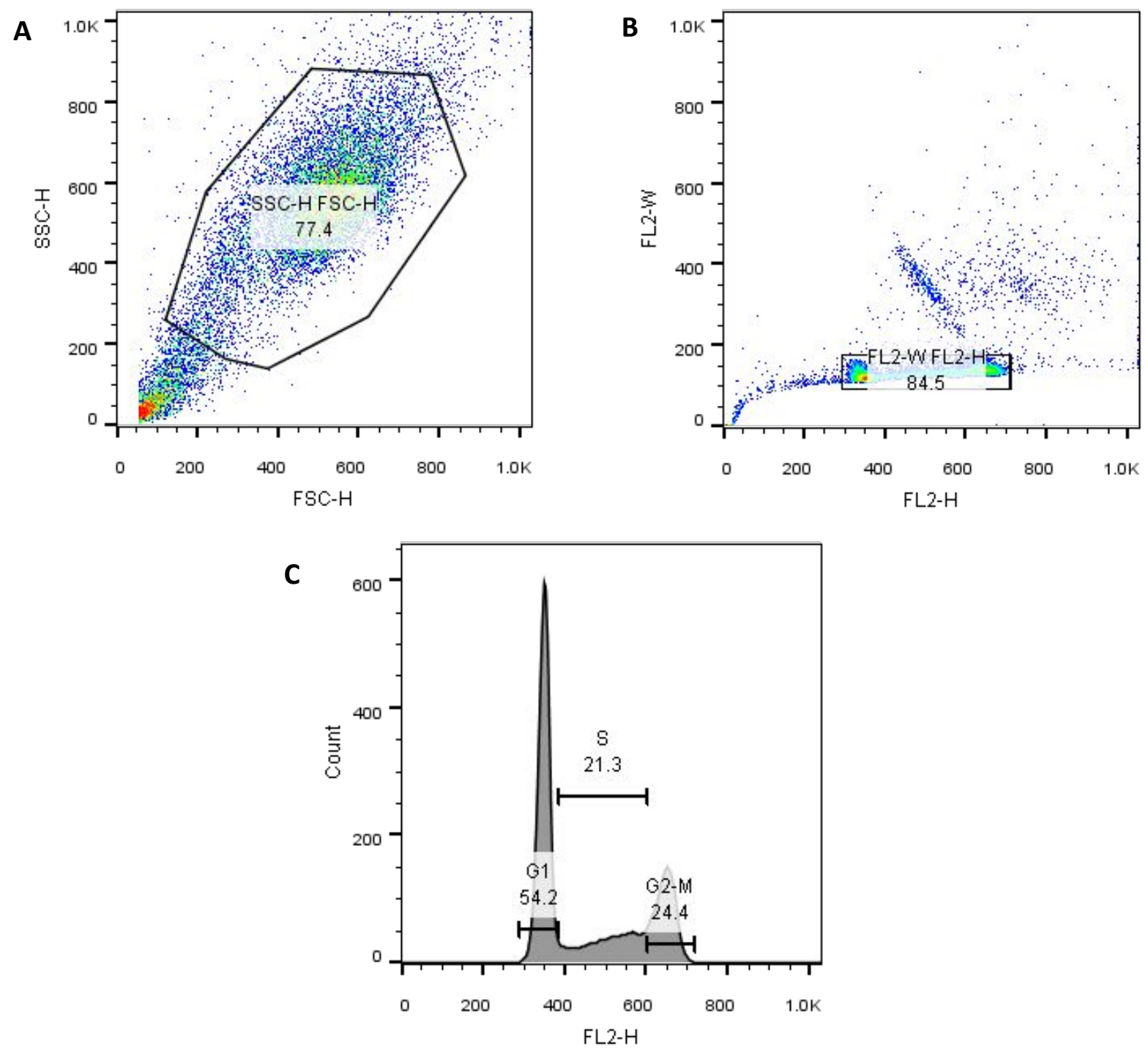

Figure SI 7. Gating method for cell cycle analysis. First gate was applied on a scatter plot (A) to gate out debris. As the cells were identified, a second gate was applied to the single cell population using pulse processing (pulse width vs. pulse area) (B) to exclude the cell doublets from analysis. From this cell population, the percentage of each subpopulation in each phase was determined by a histogram plot $(\mathbf{C})$. 